\title{
Buffered lidocaine 1\%/epinephrine 1:100,000 with sodium bicarbonate (sodium hydrogen carbonate) in a 3:1 ratio is less painful than a 9:1 ratio: A double-blind, randomized, placebo-controlled, crossover trial
}

Vent, Alexandra ; Surber, Christian ; Graf Johansen, Nicole Tracy ; Figueiredo, Verena ; Schönbächler, Georg ; Imhof, Laurence ; Buset, Caroline ; Hafner, Jürg

\begin{abstract}
Background: Neutralizing (buffering) lidocaine 1\%/epinephrine 1:100,000 solution (Lido/Epi) with sodium hydrogen carbonate (NaHCO3) (also called sodium bicarbonate) is widely used to reduce burning sensations during infiltration of Lido/Epi. Optimal mixing ratios have not been systematically investigated. Objectives: To determine whether a Lido/Epi:NaHCO3 mixing ratio of 3:1 (investigational medicinal product 1) causes less pain during infiltration than a mixing ratio of 9:1 (IMP2) or unbuffered Lido/Epi (IMP3). Methods: Double-blind, randomized, placebo-controlled, crossover trial $(\mathrm{n}=2 \times 24)$ with 4 investigational medicinal products (IMP1-4). Results: The 3:1 mixing ratio was significantly less painful than the 9:1 ratio $(\mathrm{P}=.044)$. Unbuffered Lido/Epi was more painful than the buffered Lido/Epi $(\mathrm{P}=.001$ vs IMP1; $\mathrm{P}=.033$ vs IMP2). IMP4 ( $\mathrm{NaCl} 0.9 \%$ [placebo]) was more painful than any of the anesthetic solutions $(\mathrm{P}=.001$ vs IMP1; $\mathrm{P}=.001$ vs IMP2; $\mathrm{P}=.016$ vs IMP3). In all cases, the anesthesia was effective for at least 3 hours. Limitations: Results of this trial cannot be generalized to other local anesthetics such as prilocaine, bupivacaine, or ropivacaine, which precipitate with NaHCO3 admixtures. Conclusions: Lido/Epi-NaHCO3 mixtures effectively reduce burning pain during infiltration. The 3:1 mixing ratio is significantly less painful than the 9:1 ratio. Reported findings are of high practical relevance, given the extensive use of local anesthesia today.
\end{abstract}

DOI: https://doi.org/10.1016/j.jaad.2019.09.088

Posted at the Zurich Open Repository and Archive, University of Zurich

ZORA URL: https://doi.org/10.5167/uzh-188150

Journal Article

Accepted Version

Originally published at:

Vent, Alexandra; Surber, Christian; Graf Johansen, Nicole Tracy; Figueiredo, Verena; Schönbächler, Georg; Imhof, Laurence; Buset, Caroline; Hafner, Jürg (2020). Buffered lidocaine 1\%/epinephrine 1:100,000 with sodium bicarbonate (sodium hydrogen carbonate) in a 3:1 ratio is less painful than a 9:1 ratio: A double-blind, randomized, placebo-controlled, crossover trial. Journal of the American Academy of Dermatology, 83(1):159-165.

DOI: https://doi.org/10.1016/j.jaad.2019.09.088 


\title{
Buffered lidocaine 1\%/epinephrine 1:100,000 with sodium bicarbonate (sodium hydrogen carbonate) in a $3: 1$ ratio is less painful than a 9:1 ratio: A double-blind, randomized, placebo-controlled, crossover trial
}

\author{
Alexandra Vent, MD, ${ }^{a}$ Christian Surber, Dr phil nat, ${ }^{a}$ Nicole Tracy Graf Johansen, Dr phil nat, ${ }^{b}$ \\ Verena Figueiredo, MSc, ${ }^{c}$ Georg Schonb€€achler, Dr sc nat, ${ }^{d}$ Laurence Imhof, MD, ${ }^{a}$ \\ Caroline Buset, MD, ${ }^{a}$ and Jurg€ Hafner, MD ${ }^{a}$ \\ Zurich, Winterthur, and Schlieren, Switzerland
}

Background: Neutralizing (buffering) lidocaine 1\%/epinephrine 1:100,000 solution (Lido/Epi) with sodium hydrogen carbonate $\left(\mathrm{NaHCO}_{3}\right)$ (also called sodium bicarbonate) is widely used to reduce burning sensations during infiltration of Lido/Epi. Optimal mixing ratios have not been systematically investigated.

Objectives: To determine whether a Lido/Epi: $\mathrm{NaHCO}_{3}$ mixing ratio of 3:1 (investigational medicinal product [1]) causes less pain during infiltration than a mixing ratio of 9:1 (IMP2) or unbuffered Lido/Epi (IMP3).

Methods: Double-blind, randomized, placebo-controlled, crossover trial $(n=2 \quad 3$ 24) with 4 investigational medicinal products (IMP1-4).

Results: The 3:1 mixing ratio was significantly less painful than the 9:1 ratio $(P=.044)$. Unbuffered Lido/Epi was more painful than the buffered Lido/Epi $(P=.001$ vs IMP1; $P=.033$ vs IMP2). IMP4 $(\mathrm{NaCl} 0.9 \%$ [placebo]) was more painful than any of the anesthetic solutions ( $\mathrm{P}=.001 \mathrm{vs}$ IMP1; $\mathrm{P}=$ .001 vs IMP2; $\mathrm{P}=.016 \mathrm{vs}$ IMP3). In all cases, the anesthesia was effective for at least 3 hours.

Limitations: Results of this trial cannot be generalized to other local anesthetics such as prilocaine, bupivacaine, or ropivacaine, which precipitate with $\mathrm{NaHCO}_{3}$ admixtures.

Conclusions: $\mathrm{Lido} / \mathrm{Epi}-\mathrm{NaHCO}_{3}$ mixtures effectively reduce burning pain during infiltration. The 3:1 mixing ratio is significantly less painful than the 9:1 ratio. Reported findings are of high practical relevance, given the extensive use of local anesthesia today. ( J Am Acad Dermatol https://doi.org/10.1016/j.jaad.2019.09.088.)

Key words: 3:1; admixture; bicarbonate; burning sensation; controlled; double-blind; epinephrine;

lidocaine; local anesthesia; placebo; randomized; ratio; sodium hydrogen carbonate; trial; unbuffered.

Lidocaine, an anesthetic of the amide class, is one of the most commonly used local anesthetics. It is available in a variety of concentrations $(0.5 \%-2.5 \%)$. A concentration of $1 \%$,

From the Department of Dermatology, University Hospital of Zurich $^{\mathrm{a}}$; Graf Biostatistics, Winterthur ; Hospital Pharmacy of the University Hospital Zurich, Schlieren ${ }^{\mathrm{C}}$; and Institute of Psychology, University of Zurich. ${ }^{d}$

Funding sources: Supported by the JH Rahn Foundation and Bruno Bloch Foundation.

Conflicts of interest: None disclosed.

IRB approval status: This study has been approved by the local ethical committee (KEK-ZH, Nr2015-0531) and by Swissmedic. with or without epinephrine, is the most commonly used. Epinephrine is added at a concentration of 1:100,000; it causes vasoconstriction, resulting in less bleeding, longer action, and less systemic toxicity.

Accepted for publication September 12, 2019.

Correspondence to: Jurg€ Hafner, MD, Department of Dermatology, Gloriastrasse 31, CH-8091 University Hospital of Zurich, Switzerland. E-mail: juerg.hafner@usz.ch.

Published online ddd.

0190-9622/\$36.00

2020 by the American Academy of Dermatology, Inc. https://doi.org/10.1016/j.jaad.2019.09.088 
To aid manufacture and stability, commercial lidocaine products with or without epinephrine have a pH of 2.5 to $4.0 .^{2-4}$ Acidity is assumed to be responsible for the burning sensation during infiltration. ${ }^{5}$ Seventeen peer-reviewed studies have confirmed significant pain reduction during infiltration of lidocaine when sodium hydrogen carbonate $\left(\mathrm{NaHCO}_{3}\right.$, also called sodium bicarbonate) $8.4 \%$ was added in various mixing ratios (10:1 to $5: 1)$ to buffer the solution at a neutral, more physiologic pH. ${ }^{1,6}$ All studies consistently reported that buffering did not reduce or shorten the anesthetic effect.

Based on this information, it has become common to mix lidocaine $1 \%$ /epinephrine 1:100,000 (Lido/Epi) with $\mathrm{NaHCO}_{3}$ at a 9:1 ratio (9 mL Lido/Epi plus $1 \mathrm{~mL}$

\section{CAPSULE SUMMARY}

Admixture of sodium hydrogen carbonate significantly reduces the strong burning sensation during infiltration of lidocaine $1 \%$ with epinephrine $(1: 100,000)$. The recommended ratios vary from $5: 1$ to 10:1.

This double-blind, randomized, placebocontrolled, crossover trial shows the superiority of a 3:1 mixing ratio over a 9:1 ratio or unbuffered lidocaine.
$\mathrm{NaHCO}_{3} 8.4 \%$ ). In daily prac-

tice, however, many patients still report distressing pain during infiltration. Therefore, we empirically extended mixing ratios and found that a 3:1 ratio led to virtually painless infiltrations.

To scientifically substantiate our observation, we conducted a phase 2, monocentric, double-blind, randomized, placebo-controlled crossover trial to assess pain during infiltration of 2 solutions with different ratios of Lido/Epi: $\mathrm{NaHCO}_{3}$ (3:1 and 9:1), unbuffered Lido/Epi, and sodium chloride ( $\mathrm{NaCl})$ 0.9\% (placebo).

\section{MATERIALS AND METHODS \\ Approvals}

The study was approved by the local ethical committee (KEK-ZH, Nr.2015-0531) and by Swissmedic, the national supervisory authority for drugs and medical products in Switzerland. Written informed consent was obtained from all volunteers. The trial was registered at www.clinicaltrial.gov (NCT03110393).

\section{Volunteers}

A total of 48 healthy volunteers were included and distributed to 2 groups. In each group, every volunteer was randomly allocated to receive injections in a different order (Fig 1).

Inclusion criteria were age 18 to 75 years, proficiency in German, and sufficient intellectual and linguistic abilities to fully understand and follow all trial procedures and instructions.

Exclusion criteria were hypersensitivity or allergies to local anesthetics of the amide type or to auxiliary substances such as sulfites, pregnancy (confirmed with testing), damaged skin on the arms, or inability to give informed consent.

Investigational medicinal products

Four investigational medicinal products (IMP1-4) were prepared and labeled according to a packaging and randomization plan by the hospital pharmacy of the University Hospital Zurich, according to current good manufacturing practice. To ensure a blind test and to guarantee product conformity/stability on the day of injection, IMPs 1 through 4 were prepared as sets of 2 identical vials $(5 \mathrm{~mL}): 1$ containing $\mathrm{NaHCO}_{3}$ and 1 containing Lido/Epi in appropriate concentrations. For further details, see Fig 2. The mixing took place within

1 minute before infiltration. This procedure was explicitly chosen to guarantee product conformity/stability.

\section{Injection sites and injection procedure}

Group 1 received 2 infiltrations. The injection sites were on the palmar aspect of the right and left forearms.

Group 2 received 4 infiltrations into the palmar aspect of the forearms. The injection sites were the radial portion of the palmar aspect of the right forearm, the ulnar portion of the palmar aspect of the right forearm, the ulnar portion of the palmar aspect of the left forearm, and the radial portion of the palmar aspect of the left forearm.

All sites were $5 \mathrm{~cm}$ distal from the cubital fossa. Five seconds after skin puncture, $2 \mathrm{~mL}$ of the IMP was slowly infiltrated into the superficial subcutis over a period of $15 \mathrm{~s}$ with a 30-gauge needle. The residual $8 \mathrm{~mL}$ in the syringe was discarded. The injections were performed by the same study physician throughout the investigation. The mixed study medication was at room temperature.

$\mathrm{pH}$ and osmolality of IMPs

The $\mathrm{pH}$ of the IMPs was determined with a Titrando 906 potentiometer (Metrohm, Herisau, Switzerland), according to European Pharmacopoeia 2.2.3. Osmolality of the IMPs was determined with the Advanced-3320 micro-osmometer (Advanced Instruments, Norwood MA) according to European Pharmacopoeia 2.2.35. 
Abbreviations used:

IMP: investigational medicinal product

Lido/Epi: lidocaine 1\%/epinephrine 1:100,000

$\mathrm{NaCl}$ : sodium chloride

$\mathrm{NaHCO}_{3}$ : sodium hydrogen carbonate (also

NRS: numeric rating scale

Measuring parameters and hypotheses

The following 3 measurements were recorded: pain during infiltration, patient comfort during infiltration, and duration of local anesthesia (numbness).

Quantitative rating of pain during infiltration was recorded on a 10-point numeric rating scale (NRS) immediately after infiltration of the study solution (a few seconds after removing the needle). For each volunteer, data were recorded on a sheet of paper with a NRS (from 0 , no pain to 10 , unacceptable pain). ${ }^{7}$

Qualitative rating of patient comfort during infiltration was recorded by using a sheet of paper with a choice of 4 categorical terms: desirable

(wunschenswert€), acceptable (akzeptabel ), less acceptable (weniger akzeptabel), and almost or totally unacceptable (kaum oder gar nicht akzeptabel ), which could be ticked off by the volunteers. This was done directly after recording the pain during infiltration (NSR) measurement.

The duration of local anesthesia (numbness) was recorded after infiltration (after recording the pain during infiltration and patient comfort during infiltration) within 3 to 5 minutes of removing of the needle and at 30-minute intervals up to 3 hours, each time using a standardized laser stimulus that left the skin barrier intact (erbium:glass nonablative fractional laser: $1540 \mathrm{~nm}$; 10-mm tip; fluence, $30 \mathrm{~mJ}$; pulse width, $15 \mathrm{~ms}$; Cynosure Inc, Langen, Germany). ${ }^{8}$ Numbness was recorded as present (yes) or absent (no).

The following hypotheses were tested for pain during infiltration:

Group 1 (primary endpoint):

d IMP 1 (Lido/Epi:NaHCO 3 ratio, 3:1) causes less pain during infiltration than IMP 2 (Lido/ Epi: $\mathrm{NaHCO}_{3}$ ratio, 9:1).

Group 2 (secondary endpoints):

d IMP 1 (Lido/Epi:NaHCO 3 ratio, 3:1) causes less pain during infiltration than IMP 3 (unbuffered Lido/Epi) and IMP4 (placebo).

d IMP 2 (Lido/Epi:NaHCO 3 ratio, 9:1) causes less pain during infiltration than IMP 3 (unbuffered Lido/Epi) and IMP4 (placebo).

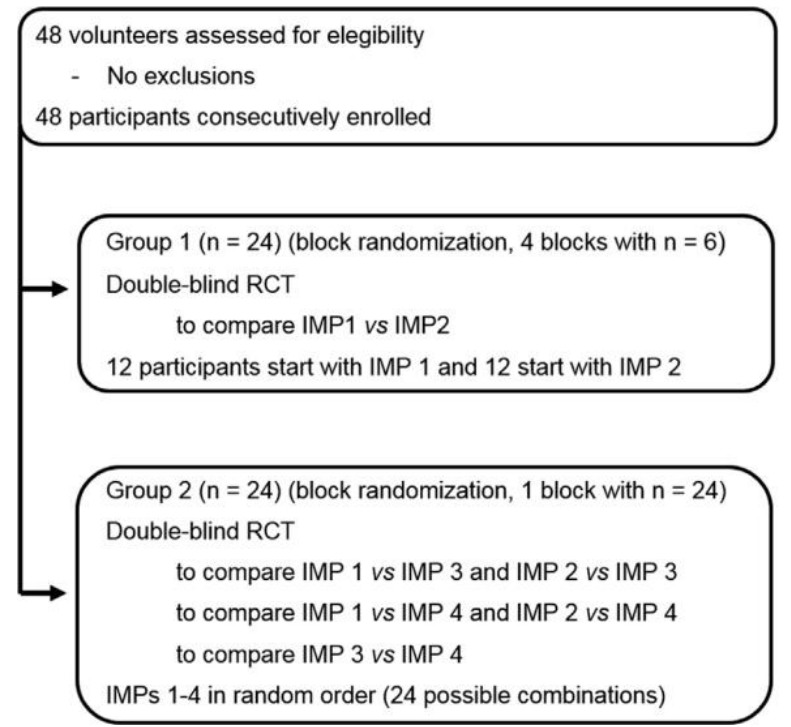

Fig 1. Consolidated Standards of Reporting Trials (CONSORT) study flow diagram. IMP 1: Lido/Epi: $\mathrm{NaHCO}_{3}$ ratio, 3:1; IMP 2: Lido/Epi: $\mathrm{NaHCO}_{3}$ ratio, 9:1; IMP 3: Lido/Epi without $\mathrm{NaHCO}_{3}$. IMP 4: $\mathrm{NaCl}$ 0.9\% (placebo). IMP, Investigational medicinal product; Lido/Epi, lidocaine 1\%/epinephrine 1:100,000; $\mathrm{NaCl}$, sodium chloride; $\mathrm{NaHCO}_{3}$, sodium bicarbonate; $\mathrm{RCT}$, randomized controlled trial.

\section{Statistics}

Group 1, primary endpoint (n 5 24). We planned for a 2-sided Mann-Whitney test of the Collaboration in Research and Methodology for Official Statistics estimator, measured as the score for pain on infiltration of the first injection minus the score for pain on infiltration of the second injection. We estimated that 24 crossovers would be needed to have at least $80 \%$ power with significance testing at the $\mathrm{a}=.05$ level to detect a difference between the 2 randomization groups (2 different orders of the injection sequence) of 3 NRS values, assuming a standard deviation of 2.5. Variability was estimated based on a 2010 Cochrane review. ${ }^{6}$

Group 2, secondary endpoints ( $\mathrm{n} 5$ 24). We decided to test all 4 IMPs on a second group of volunteers to avoid possible interference with the investigation of the primary endpoint (IMP 1 vs IMP 2). Every volunteer of group 2 was randomly allocated a different order of injections.

The randomization list was computer generated by the hospital pharmacy, with block sizes of 6 for group 1 (4 blocks of 6 participants; 3 started with IMPs 1 and 3 with IMP 2) and 1 block of 24 participants for group 2 (this block contained 24 possible combinations).

The tests for differences in pain during infiltration are based on a 2-sided exact MannWhitney test of the CROS-estimator. 


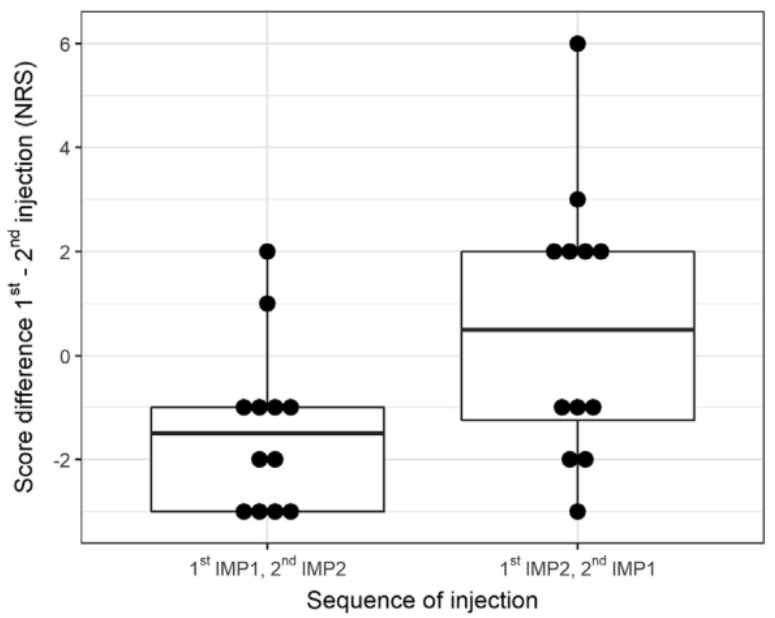

Fig 3. Quantitative rating of pain during infiltration, IMP 1 versus IMP 2. Group $1(n=24)$, primary endpoint. The volunteers of group 1 compared IMP 1 versus IMP 2 in a randomized sequence (block randomization, 4 blocks of 6 volunteers). IMP 1 was significantly less painful during infiltration than IMP $2(P=.044)$. When IMP 1 was followed by IMP 2, IMP 1 had a median pain score 1.5 points lower (less painful) than IMP 2. When IMP 2 was followed by IMP 1, the IMP 1 median pain score was 0.5 points lower than for IMP 2 .

In the boxplots, the line in the box corresponds to the median; the lower and upper hinges correspond to the first and third quartiles, respectively; and the upper and lower whiskers extend from the hinge to the largest or smallest value, respectively, no further than $1.53 \mathrm{IQR}$ from the hinge. The dots show each of the 24 NRS score points. IMP

1: Lido/Epi: $\mathrm{NaHCO}_{3}$ ratio, 3:1; IMP 2: Lido/Epi: $\mathrm{NaHCO}_{3}$ ratio, 9:1. IMP, Investigational medicinal product; IQR, interquartile range; Lido/Epi, lidocaine 1\%/epinephrine 1:100,000; $\mathrm{NaHCO}_{3}$, sodium bicarbonate; NRS, numeric rating scale.

\section{DISCUSSION}

In a phase 2, monocentric, double-blind, random-ized, placebo-controlled, crossover trial, we scientifically substantiated that the mixing of Lido/Epi: $\mathrm{NaHCO}_{3}$ at a 3:1 ratio causes less pain during infiltration than at a 9:1 ratio (Fig 3). Furthermore, we showed that unbuffered Lido/Epi is more painful than buffered Lido/Epi at ratios of 3:1 and 9:1 and that the placebo was more painful than any of the anesthetic solutions (Fig 4). Patient comfort during infiltration, assessed with qualitative pain scores, showed a clear preference for the buffered Lido/Epi at a ratio of 3:1. Placebo received the most negative scores (Fig 5). All anesthetic solutions led to numbness in the injection areas between 5 minutes and 3 hours after infiltration. The lower lidocaine concentration in the 3:1 Lido/Epi: $\mathrm{NaHCO}_{3}$ did not affect local anesthesia, compared with the 9:1 ratio, within the observation time.

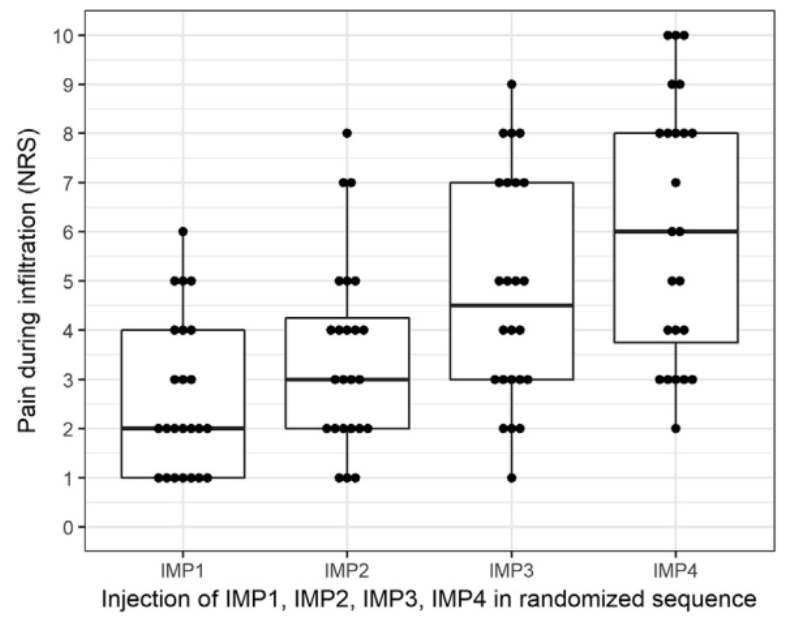

Fig 4. Quantitative rating of pain during infiltration: IMP 1 versus IMP3 and IMP4; IMP2 versus IMP3 and IMP 4. Group $2(n=24)$, secondary endpoint. The volunteers of group 2 compared IMP 1 versus IMP3 and IMP4 and compared IMP 2 versus IMP3 and IMP4 in a randomized sequence (block randomization, 1 block of 24 different combinations). Statistical significance was as follows: IMP 1 versus IMP $3, \mathrm{P}=.001$; IMP 2 versus IMP $3, \mathrm{P}=.033$; IMP 1 versus IMP 4, P = .001; IMP 2 versus IMP 4, P = .001 ; IMP 3 versus IMP $4, \mathrm{P}=.016$.

In the boxplots, the line in the box corresponds to the median; the lower and upper hinges correspond to the first and third quartiles, respectively; and the upper and lower whiskers extend from the hinge to the largest or smallest value, respectively, no further than $1.53 \mathrm{IQR}$ from the hinge. The dots show each of the 24 NRS score points. IMP

1: Lido/Epi: $\mathrm{NaHCO}_{3}$ ratio, 3:1; IMP 2: Lido/Epi: $\mathrm{NaHCO}_{3}$ ratio, 9:1. IMP, Investigational medicinal product; IQR, interquartile range; Lido/Epi, lidocaine 1\%/epinephrine 1:100,000; $\mathrm{NaHCO}_{3}$, sodium bicarbonate; NRS, numeric rating scale.

Acidity causes the burning sensation during infiltration

Acidity has been assumed to be responsible for the burning sensation during infiltration, and the detection of acid-sensing ion channel receptors or nociceptors fully supports this explanation. ${ }^{5}$ The causal link between $\mathrm{pH}$ and burning pain during infiltration is also supported by our trial with the unbuffered Lido/Epi solution (IMP 3) at pH 3.8 and with the pharmacologically inactive placebo (IMP 4) at $\mathrm{pH}$ 6.3. The pain during infiltration with the neutralized solutions (IMP 1, pH 7.5; IMP 2, pH 7.3) was significantly reduced.

At neutral $\mathrm{pH}$, lidocaine is predominantly present in its active form

Injection solutions contain lidocaine in an uncharged, nonionized form and in a charged, ionized form. The uncharged formdalso known as the active formdis lipophilic and, in contrast to the 


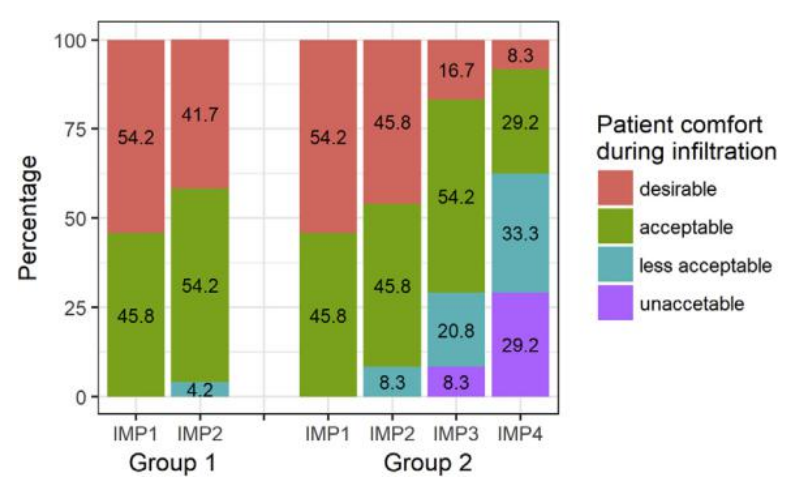

Fig 5. Qualitative rating of patient comfort during infiltration of local anesthetics, captured with categorial terms. The first 2 bars represent results from group 1 , and the next 4 bars represent results from group 2. IMP 1: Lido/Epi: $\mathrm{NaHCO}_{3}$ ratio, 3:1; IMP 2: Lido/Epi:NaHCO 3 ratio, 9:1. IMP, Investigational medicinal product; Lido/Epi, lidocaine 1\%/epinephrine 1:100,000; NaHCO3, sodium bicarbonate.

charged (hydrophilic) form, readily permeates the nerve membrane to bind from the cytosol to the acid-sensing ion channel receptors. ${ }^{10,11}$ According to the Henderson-Hasselbalch equation, in any sample of a lidocaine solution, the ratio of the nonionized (active form) to ionized species of the anesthetic depends on the $\mathrm{pH}$. At a more acidic $\mathrm{pH}$, the ionized cationic form predominates. For instance, at a $\mathrm{pH}$ of 3.8 , a typical cartridge of Lido/Epi contains only 1 molecule of nonionized (active form) anesthetic for every 10,000 molecules of ionized anesthetic. On the other hand, closer to physiologic $\mathrm{pH}$, more nonionized (active form) anesthetic is present. For instance, at the physiologic $\mathrm{pH} 7.4$, there is 1 molecule of nonionized (active form) lidocaine in solution for every 4 molecules of ionized lidocaine. At the physiologic $\mathrm{pH}, 2500$ times more of the active form is available than at a pH of 3.8.

$\mathrm{NaCl} 0.9 \%$ is more painful than unbuffered Lido/Epi

During injection of unbuffered Lido/Epi ( $\mathrm{pH} 3.8)$, the buffering system of the body will bringdwith a very short time lagdthe nonphysiologic $\mathrm{pH}$ of the solution to a more neutral level. ${ }^{12}$ During neutralization, the continuously formed nonionized lidocaine (active form) can penetrate nerve cells and block the acid-sensing ion channel receptors from inside the synapse. ${ }^{13,14}$ With $\mathrm{NaCl} 0.9 \%$ (pH 6.3), the buffering system of the body will also neutralize the solution. However, due to the absence of lidocaine, acidity will cause a noticeably longer duration of burning sensation until neutralization of the solution.
Carbon dioxide has analgesic effects

When $\mathrm{NaHCO}_{3}$ is mixed with acidic lidocaine hydrochloride solution, water $\left(\mathrm{H}_{2} \mathrm{O}\right)$ and carbon dioxide $\left(\mathrm{CO}_{2}\right)$ are formed. Condouris and Shakalis ${ }^{15}$ showed that $\mathrm{CO}_{2}$ develops an independent, direct local anesthetic effect. Based on these observations, Catchlove was able to demonstrate that $\mathrm{CO}_{2}$ enhances the action of lidocaine. $\mathrm{CO}_{2}$ directly deactivates the nerve axon and indirectly increases the anesthetic effect of lidocaine by changing its electrical charge. ${ }^{16}$ This may explain why an excess of $\mathrm{CO}_{2}$, as formed in a 3:1 mixture, more effectively reduces the burning sensation of lidocaine relative to the 9:1 mixture. Both mixtures effectively neutralize the Lido/Epi solution, but the 3:1 mixture provides more local $\mathrm{CO}_{2}$ anesthesia.

Osmolality and pain during infiltration Osmolality may impair tolerability (including pain

on infiltration) of injection solutions. ${ }^{17}$ The general osmolality recommendation for injection and infusion solutions is to not greatly exceed 600 and 1000 $\mathrm{mOsm} / \mathrm{kg}$, respectively. ${ }^{17}$ No $\mathrm{mOsm} / \mathrm{kg}$-to-pain relationship was found between 300 and 1100 $\mathrm{mOsm} / \mathrm{kg}$ in a study after intramuscular injection of vaccine suspensions in healthy adults. ${ }^{18}$ The osmolality of Lido/Epi: $\mathrm{NaHCO}_{3}$ at a ratio of 3:1 (IMP 1) was $674.6 \mathrm{mosm} / \mathrm{kg}$ and higher than in the other solutions (467.1, 315.0, and $285.3 \mathrm{mosm} / \mathrm{kg}$ for IMPs 1, 2, and 3, respectively). In a similar investigation, Parham and Pasieka ${ }^{19}$ found that pain during infiltration of physiologic $\mathrm{NaCl} 0.9 \%$ (285.3 $\mathrm{mosm} / \mathrm{kg}$ ) was significantly more painful than Lido/Epi-NaHCO 3 mixtures that were more than twice as osmolar. ${ }^{19}$ Our data confirm this observation.

Stability of bicarbonate-neutralized Lido/Epi solution

The stability of neutralized Lido/Epi solutions with $\mathrm{NaHCO}_{3}$ is limited, ${ }^{20}$ which is the major reason why there are no commercial products on the market. Neutralized Lido/Epi solutions are compounded in advancedeither preoperatively by nurses or physicians or by a specialized pharmacy. ${ }^{20}$ The manner in which the solutions are mixed often depends on the available bulk containers (drug/buffer), syringes, and local situations and customs. This makes the storage and subsequent stability of the compounded product an important issue. Despite numerous examinations on the stability of neutralized anesthetics/epinephrine solutions, there is still a considerable degree of uncertainty on the topic. Due to the lack of proper 
method validation and study design and the large number of influences when compounding (such as temperature, time, packaging material, light, and oxygen level), no general rules have emerged to simplify compounding and ensure product quality. In the context of our trial, we therefore limited the time between mixing and infiltrating the Lido/Epi- $\mathrm{NaHCO}_{3}$ solution to 1 minute so that degradation would not be a factor. In our everyday practice, we limit the shelf life of the ad hoceprepared Lido/Epi- $\mathrm{NaHCO}_{3}$ solutions to the duration of the surgical intervention (12 hours).

\section{CONCLUSIONS}

The Lido/Epi-NaHCO 3 mixtures effectively reduce burning pain during infiltration. The 3:1 mixing ratio was significantly less painful than the 9:1 ratio. The reported findings are of high practical relevance, given the extensive use of local anesthesia today.

\section{Limitations}

The results of this clinical trial cannot be generalized to other local anesthetics, such as prilocaine, bupivacaine, or ropivacaine, which precipitate with bicarbonate admixture. $\mathrm{NaHCO}_{3}$ admixtures with other concentrations of lidocaine (eg, $0.5 \%$ or $2.0 \%$ ) and with lidocaine without epinephrine are expected to function the same way, but this has not been formally tested.

This study was supported by the JH Rahn Foundation, Zurich (www.jhrahn-foundation.ch) and the Bruno Bloch Foundation, Zurich (www.brunoblochstiftung.ch). We thank all participants.

\section{REFERENCES}

1. Burns CA, Ferris G, Feng C, et al. Decreasing the pain of local anesthesia: a prospective, double-blind comparison of buffered, premixed $1 \%$ lidocaine with epinephrine versus $1 \%$ lidocaine freshly mixed with epinephrine. J Am Acad Dermatol. 2006;54:128-131.

2. Christoph RA, Buchanan L, Begalla K, Schwartz S. Pain reduction in local anesthetic administration through $\mathrm{pH}$ buffering. Ann Emerg Med. 1988;17:117-120.
3. Masters JE. Randomised control trial of $\mathrm{pH}$ buffered lignocaine with adrenaline in outpatient operations. $\mathrm{Br} \mathrm{J}$ Plast Surg. 1998; 51:385-387.

4. Crews JC, Clark RB. Effect of alkalinization on the $\mathrm{pH}$ of local anesthetic solutions. Anesth Analg. 1987;66:1203.

5. Yoder N, Yoshika C, Gouaux E. Gating mechanisms of acidsensing ion channels. Nature. 2018;555(7696):397-401.

6. Cepeda MS, Tzortzopoulou A, Thackrey M, et al. Adjusting the $\mathrm{pH}$ of lidocaine for reducing pain on injection. Cochrane Database Syst Rev. 2010;(12):CD006581.

7. Miro J, Castarlenas E, de la Vega R, et al. Validity of three rating scales for measuring pain intensity in youths with physical disabilities. Eur J Pain. 2016;20:130-137.

8. Plaghki L, Mouraux A. How do we selectively activate skin nociceptors with a high power infrared laser? Physiology and biophysics of laser stimulation. Neurophysiol Clin. 2003;33: 269-277.

9. R: A Language and Environment for Statistical Computing [software]. Vienna, Austria: R Foundation for Statistical Computing; 2017.

10. Hille B. The $\mathrm{pH}$-dependent rate of action of local anesthetics on the node of Ranvier. J Gen Physiol. 1977;69: 475-496.

11. Hille B. Local anesthetics: hydrophilic and hydrophobic pathways for the drug-receptor reaction. J Gen Physiol. 1977; 69:497-515.

12. Gardner $\mathrm{JH}$, Semb J. The relation of $\mathrm{pH}$ and surface tension to the activity of local anesthetics. J Pharmacol Exp Ther. 1935;54: 309-319.

13. Talu $\mathrm{H}$, Elibol $\mathrm{O}$, Yanyali $A$, et al. Effect of warming and buffering lidocaine on pain during facial anesthesia. Ann Ophthalmol. 2001;33:43-47.

14. Richtsmeier AJ, Hatcher JW. Buffered lidocaine for skin infiltration prior to hemodialysis. J Pain Symptom Manage. 1995;10:198-203.

15. Condouris GA, Shakalis A. Potentiation of the nervedepressant effect of local anesthetics by carbon dioxide. Nature. 1964;204:57-58.

16. Catchlove RFH. The influence of $\mathrm{CO}_{2}$ and $\mathrm{pH}$ on local anesthetic action. J Pharmacol Exp Ther. 1972;181:298-309.

17. Wang W. Tolerability of hypertonic injectables. Int J Pharm. 2015;490:308-315.

18. Nony $P$, Girard $P$, Chabaud $S$, et al. Impact of osmolality on burning sensations during and immediately after intramuscular injection of $0.5 \mathrm{ml}$ of vaccine suspensions in healthy adults. Vaccine. 2001;19:3645-3651.

19. Parham SM, Pasieka JL. Effect of $\mathrm{pH}$ modification by bicarbonate on pain after subcutaneous lidocaine injection. Can J Surg. 1996;39:31-35.

20. Pascuet E, Donnelly RF, Garceau D, et al. Buffered lidocaine hydrochloride solution with and without epinephrine: stability in polypropylene syringes. Can J Hosp Pharm. 2009; 62:375-380. 